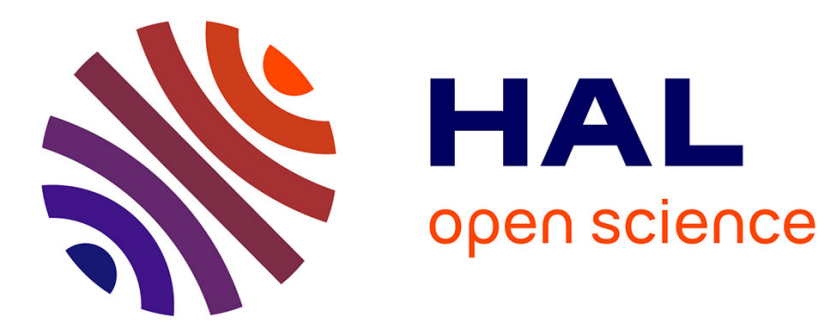

\title{
Le BPR fibré sous sollicitation biaxiale
}

Esteban Astudillo, Gérard Bernier, Olivier Ple

\section{To cite this version:}

Esteban Astudillo, Gérard Bernier, Olivier Ple. Le BPR fibré sous sollicitation biaxiale. Revue Française de Génie Civil , 2002, 6 (5), pp.709-722. 10.1080/12795119.2002.9692397 . hal-00484252

\section{HAL Id: hal-00484252 \\ https://hal.science/hal-00484252}

Submitted on 18 May 2010

HAL is a multi-disciplinary open access archive for the deposit and dissemination of scientific research documents, whether they are published or not. The documents may come from teaching and research institutions in France or abroad, or from public or private research centers.
L'archive ouverte pluridisciplinaire HAL, est destinée au dépôt et à la diffusion de documents scientifiques de niveau recherche, publiés ou non, émanant des établissements d'enseignement et de recherche français ou étrangers, des laboratoires publics ou privés. 


\title{
Le BPR fibré sous sollicitation biaxiale
}

\section{Étude expérimentale du comportement mécanique en bitraction du Béton de Poudres Réactives (BPR) fibré}

\author{
Esteban Astudillo, Gérard Bernier, Olivier Plé \\ Laboratoire de Mécanique et Technologie, \\ ENS de Cachan / CNRS / Université Paris 6 \\ 61 avenue du Président Wilson, F-94235 Cachan cedex \\ bernier@dgc.ens-cachan.fr,ple@dgc.ens-cachan.fr
}

\begin{abstract}
RÉSUMÉ. Nous exposons dans cet article une étude expérimentale qui permet d'identifier le comportement mécanique en bitraction du Béton de Poudres Réactives (BPR) renforcé de fibres courtes d'acier. Nous présentons d'abord la pertinence de l'étude et les caractéristiques du matériau. Nous décrivons ensuite la conception et les particularités du dispositif expérimental utilisé qui comprend la prise et le dépouillement d'images numériques. Les résultats sont finalement discutés, notamment ceux qui se rapportent aux efforts, déformations et champ de déplacement.

ABSTRACT. An experimental study which allows identifying the biaxial tensile behavior of the Reactive Powder Concrete (RPC) reinforced with short steel fibers is reported. We present first the suitability of the study and the characteristics of the building material. We expose then the conception and the peculiarities of the test setup which includes digital image correlation. Results are finally discussed, specially those that relate to strength, strains and displacement field.

MOTS-CLÉS : Béton de Poudres Réactives (BPR), essai de bitraction, corrélation d'images, champ de déplacement, déformation.

KEY WORDS: Reactive Powder Concrete (RPC), bitensile test, digital image correlation, displacement field, strain.
\end{abstract}




\section{Introduction}

Les essais uniaxiaux classiques sont souvent insuffisants pour décrire correctement le comportement du béton dans ses applications réelles. On se satisfait, dans la plupart des cas, d'extrapoler les résultats d'un essai uniaxial pour décrire le comportement multiaxial. Cela peut s'expliquer par la difficulté de mise en œuvre d'un essai multiaxial sur des matériaux de type béton. La littérature ne présente qu'un nombre restreint d'études de ce type [KUP 69][HUS 00]. D'ailleurs, les règlements constructifs ne reposent en général que sur la connaissance du comportement du béton sous sollicitations simples, tandis que celui-ci, dans ses multiples applications, est souvent soumis à des chargements complexes.

\subsection{Le Béton de Poudres Réactives (BPR) armé de fibres}

Composé de poudres (sable, ciment quartz, fumée de silice) dont la taille des plus gros grains ne dépasse pas $500 \mu \mathrm{m}$, le BPR possède une porosité très faible et un arrangement granulaire très compact, qui résultent d'une grande défloculation des particules fines obtenue par l'utilisation d'adjuvants superplastifiants (permettant une réduction de l'eau de gâchage). Ces matrices cimentaires ont un comportement purement fragile. L'ajout de fibres modifie ce comportement en conférant une certaine ductilité. Il s'agit, pour le matériau de cette étude, de fibres d'acier de $13 \mathrm{~mm}$ de longueur et $0,16 \mathrm{~mm}$ de diamètre dosées a $2 \%$ en volume. Les fibres ont pour fonction essentielle de suppléer la perte de résistance en traction de la matrice au droit des fissures [RIC 95]. Ce béton est fabriqué dans des conditions voisines de celles des bétons classiques, mais, pour atteindre ses performances maximales en terme de résistance et de durabilité, il nécessite un étuvage de $48 \mathrm{~h}$ à une température moyenne de $90^{\circ} \mathrm{C}$. Ce traitement offre de plus quelques avantages comme la quasidisparition du retrait de dessiccation. Ce béton permet d'atteindre des résistances de $180 \mathrm{MPa}$ en compression, de 7 à $12 \mathrm{MPa}$ en traction directe et d'obtenir des caractéristiques de durabilité bien supérieures à celles des bétons classiques [BEH 96]. Il faut noter que la résistance en traction de ce matériau est la propriété la plus intéressante car elle a un caractère de fiabilité indéniable. Des essais de traction uniaxiale ont déjà été réalisés [BEH 96] et nous nous bornons maintenant au cas d'une sollicitation en traction biaxiale.

\section{Mise au point de l'essai de traction biaxiale sur éprouvette cruciforme}

Ce type d'essai permet la création d'états de contraintes biaxiaux réalistes très proches de ceux observés dans les structures. Il s'agit de solliciter les quatre extrémités d'une éprouvette en croix suivant deux directions orthogonales dans le plan de l'éprouvette. Le domaine possible de contraintes est donné sur la figure 1. 


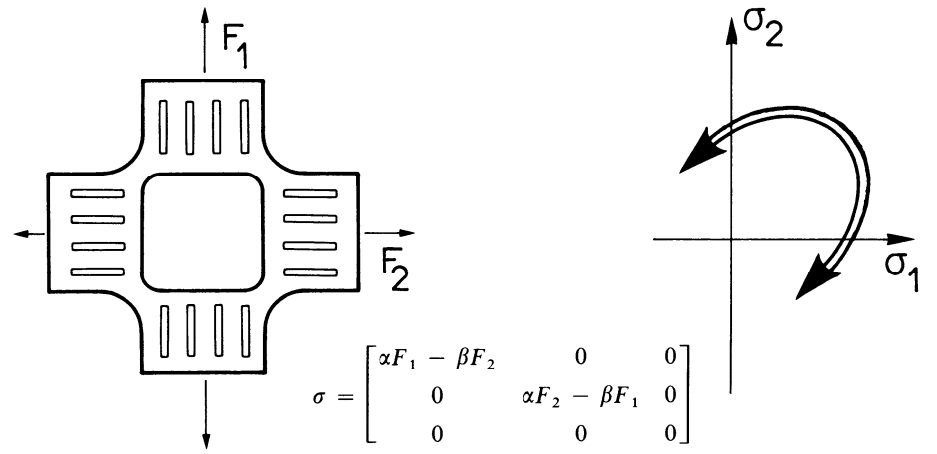

Figure 1. Essai de bitraction sur éprouvette cruciforme et domaine possible de contraintes.

La morphologie de l'éprouvette résulte du principe suivant : si on observe un modèle en éléments finis d'une plaque carrée chargée en traction biaxiale par l'intermédiaire de broches situées près du contour, l'uniformité théorique des déformations est perturbée par les déformations de Poisson empêchées sur le contour. Pour créer alors un champ de contrainte uniforme on introduit le concept d'évidement dans les bras de chargement. Ceux-ci permettant d'éloigner la zone de déformation empêchée du centre de l'éprouvette et de transmettre les efforts de traction par l'intermédiaire des peignes ainsi constitués. La géométrie des éprouvettes en croix possède donc, en général, des évidements dans les bras plus un amincissement uniforme de la partie centrale (zone utile). Cependant, les dimensions et la géométrie finales de l'éprouvette dépendent fortement du matériau, des capacités de la machine à utiliser et du type d'essai que l'on souhaite réaliser [MOR 85] [COG 97].

\subsection{La machine multiaxiale ASTREE du LMT}

La géométrie de l'éprouvette dépendant fortement de la machine d'essais utilisée, nous allons présenter en quelques mots "ASTREE", la machine multiaxiale du Laboratoire de Mécanique et Technologie (figure 2), avec laquelle nos essais de bitraction sont réalisés.

Le bâti d'ASTREE est formé d'un soubassement fixe, de quatre colonnes verticales et d'une traverse supérieure mobile. Cette machine électro-hydraulique asservie est equipée de six vérins opposés deux à deux dans trois directions orthogonales de chargement (figure 3). L'espace d'essai délimité par les vérins est de $900 \times 900 \times 1500 \mathrm{~mm}^{3}$. La capacité des quatre vérins horizontaux est de $\pm 100 \mathrm{kN}$ et de $\pm 250 \mathrm{kN}$ pour les deux verticaux. 


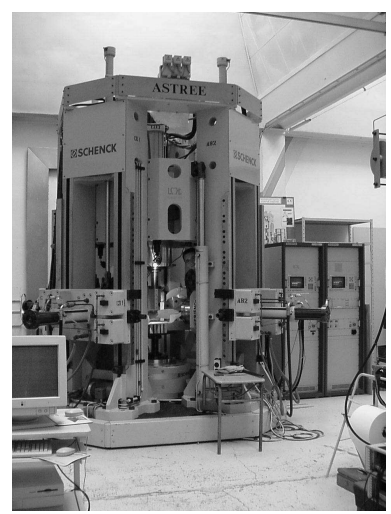

Figure 2. La machine multiaxiale ASTREE

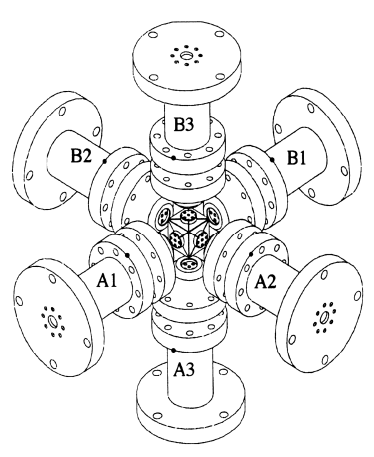

Figure 3. Disposition des vérins d'ASTREE

\subsection{L'éprouvette cruciforme en Béton de Poudres Réactives fibré}

S'il est vrai que l'essai de bitraction sur éprouvette cruciforme est le mieux adapté à nos besoins, il présente néanmoins des difficultés expérimentales particulières D'ailleurs, notre recherche bibliographique a montré qu'un tel essai sur ce type de matériau, n'avait pas encore été réalisé. En ce qui concerne le corps d'épreuve il a fallu concevoir une éprouvette répondant à divers enjeux, parmi lesquels on peut citer les suivants :

- La taille de l'éprouvette : Elle doit impérativement être en rapport avec la machine à utiliser (taille et capacité), en l'occurrence la presse multiaxiale ASTREE, ne dispose que d'un espace libre maximum entre vérins de $900 \mathrm{~mm}$. Il faut donc concevoir une éprouvette de $800 \mathrm{~mm}$ maximum, puisque dans ce même espace, le système d'accrochage machine - éprouvette y sera hébergé. Le corps d'épreuve doit également permettre d'analyser le matériau à une échelle réaliste pour être représentatif du matériau tel qu'il se trouve dans ses applications. Nous proposons alors un volume utile (partie amincie) de $100 \times 100 \times 20 \mathrm{~mm}^{3}$ afin de tester un échantillon de matériau suffisamment grand par rapport à la taille des fibres (13 $\mathrm{mm}$ ). Il faut penser d'ailleurs, que la zone utile doit permettre une instrumentation correcte vis-à-vis des techniques d'acquisition à utiliser.

- La géométrie de l'éprouvette : Elle doit répondre à la double condition de l'essai ; un champ de contraintes de bitraction uniforme dans la zone utile et une zone centrale la plus sollicitée. Globalement, notre géométrie de départ est une éprouvette cruciforme, comportant une zone utile carrée plane au centre et des peignes, dont l'épaisseur proposée cinq fois plus grande que l'épaisseur de la zone 
utile ; ce rapport d'épaisseur favorise la concentration de contraintes dans la zone utile sans risquer des ruptures prématurées dans les bras de chargement. Entre la zone utile et les peignes le raccordement est elliptique. Le raccordement de deux peignes perpendiculaires est décrit par une surface qui résulte de la rotation de l'ellipse.

\subsubsection{Optimisation de la géométrie de l'éprouvette}

La géométrie et les dimensions proposées sont ensuite optimisées à l'aide du code d'éléments finis CASTEM 2000 pour avoir, en élasticité, un champ de contraintes de traction le plus uniforme possible dans la zone utile et une zone centrale la plus sollicitée.

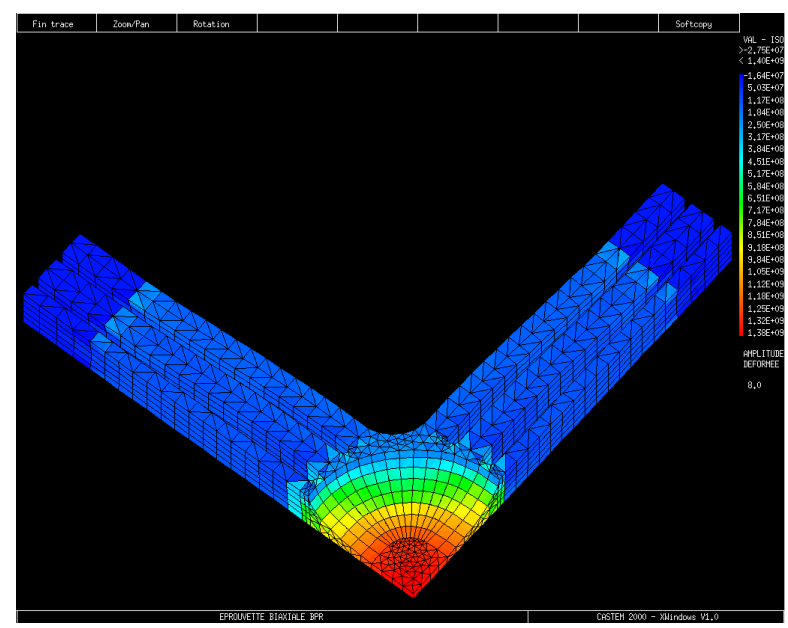

Figure 4 : Résultat final de l'optimisation

L'optimisation a été réalisée à l'aide d'un calcul en éléments finis à partir d'un maillage 3D d'éléments à quatre nœuds. Le matériau considéré comme élastique linéaire isotrope de module d'Young E=50 $000 \mathrm{Mpa}$ et de coefficient de Poisson $v=0.18$. Les conditions de symétrie ont permis de ne modéliser qu'un huitième de l'éprouvette. Le résultat final de l'optimisation de la géométrie est montré par la figure 4. Pour ce calcul on a imposé un déplacement aux extrémités de l'éprouvette, les bras étant encastrés à la machine. Les zones rouges indiquent les zones où les contraintes principales sont les plus importantes et les zones bleues les endroits où ces contraintes sont les plus faibles. Les dimensions finales de l'éprouvette sont montrées dans la figure 5 . 


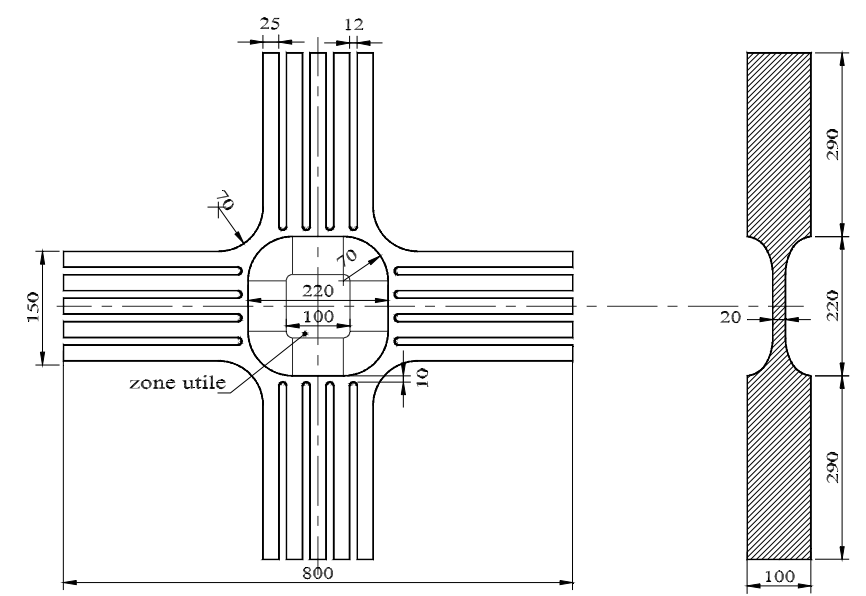

Figure 5 : Géométrie de l'éprouvette optimisée (Cotation mm)

\subsubsection{Fabrication des éprouvettes}

- Moulage des éprouvettes : Au cours de l'optimisation de l'éprouvette, nous avons décidé de faire un moulage le plus proche possible de la géométrie retenue. Le contour de l'éprouvette suit donc le moulage définitif et seule la partie centrale, où la zone utile exige la complète disparition des effets de paroi, sera usinée. Dans le cas des bras de chargement ces effets sont au contraire recherchés pour minimiser les risques de rupture en traction. Des moules en bois répondant à ces conditions ont été fabriqués. La figure 6 montre un moule fini et prêt à être utilisé.

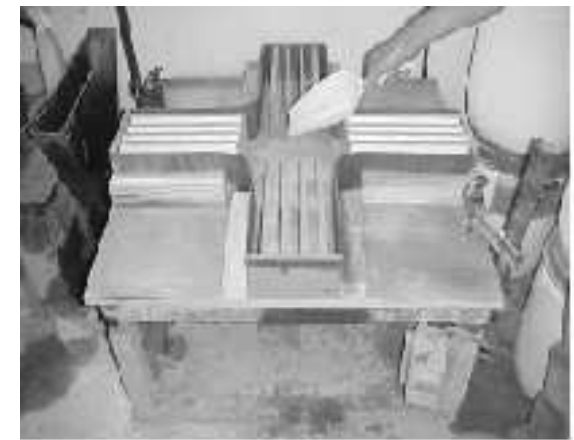

Figure 6: Moule des éprouvettes

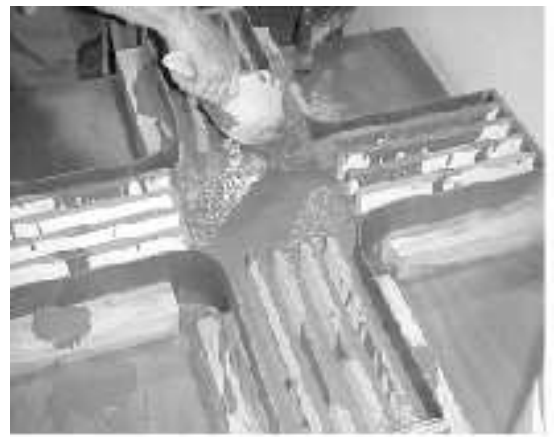

Figure 7 : Coulage des éprouvettes

- Coulage des éprouvettes : la procédure utilisée pour le remplissage des moules devait privilégier l'orientation des fibres au niveau de bras et, en même temps, éviter des effets indésirables dans la partie centrale (Figure 7). Nous avons donc procédé à un remplissage par écoulement parallèle aux bras des éprouvettes en alternant le sens 
et la direction du coulage au fur et à mesure du remplissage. Cette procédure doit induire une organisation bidirectionnelle des fibres dans la partie centrale.

- Étuvage des éprouvettes : pour atteindre ses performances maximales en terme de résistance et de durabilité, le BPR nécessite un étuvage de 48h à une température moyenne de $90^{\circ}$. Une fois démoulées, nos éprouvettes ont suivi ce traitement.

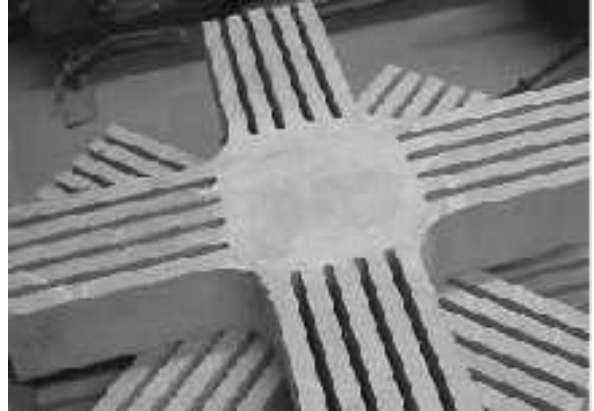

Figure 8 : Éprouvette avant usinage

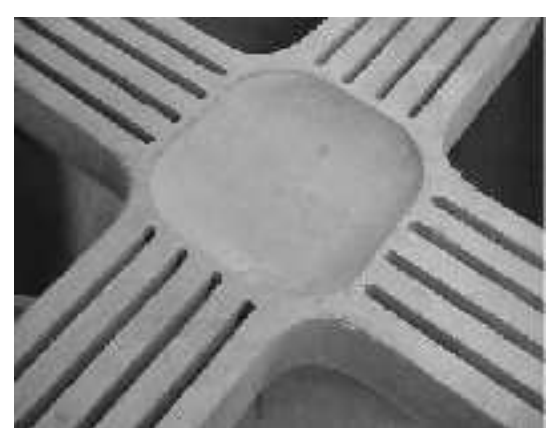

Figure 9 : Éprouvette après usinage

- Usinage des éprouvettes : Les éprouvettes fabriquées subissent sur chaque face un usinage de la partie centrale afin de correspondre à la géométrie retenue lors de la modélisation. Cet usinage est réalisé à l'aide d'un outil diamanté piloté par une machine à commande numérique, qui garanti une correcte correspondance au niveau de mesures et raccordements propres de la géométrie. Les figures 8 et 9 montrent, respectivement, une éprouvette avant et après usinage.

\section{Pilotage et acquisition de l'essai de bitraction}

L'éprouvette placée dans le plan horizontal de la machine est fixée par l'intermédiaire d'équerres métalliques boulonnées aux vérins. On vérifie avant et après collage le centrage et l'horizontalité de l'éprouvette pour s'assurer de ne pas induire des flexions parasites dans le plan ou dans la hauteur de l'éprouvette. La figure 10 montre la configuration de l'essai une fois l'éprouvette mise en place.

Les essais sont asservis en déplacement à partir des capteurs de déplacement des vérins. Plusieurs chemins de chargement ont été utilisés afin d'obtenir dans la zone utile une sollicitation de bitraction symétrique. Les essais ont montré que la symétrie de déplacements des vérins ne fournit pas forcément une symétrie de déformations dans la zone utile. 


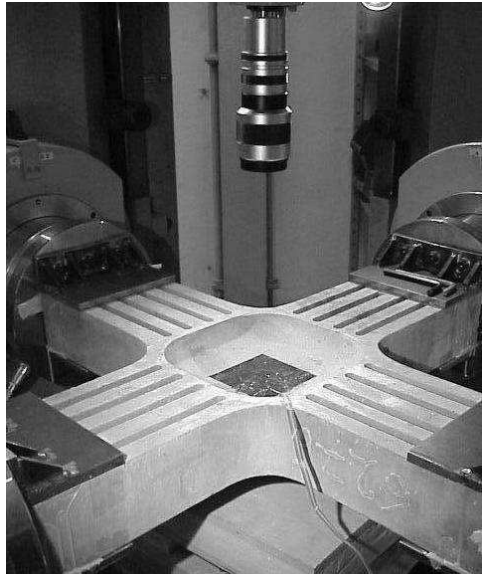

Figure 10 : Configuration essai

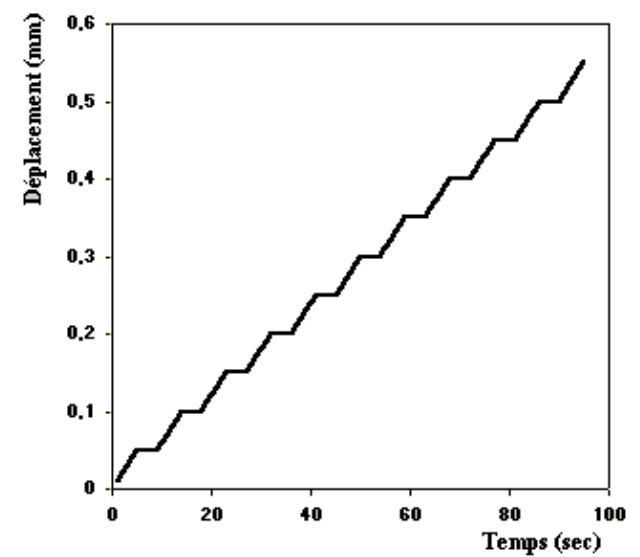

Figure 11 : Chargement appliqué

\subsection{Instrumentation}

Les corps d'épreuve ont été équipés de jauges de déformation et suivis optiquement par analyse d'images. Trois jauges de déformations de $30 \mathrm{~mm}$ superposées à $0^{\circ}, 45^{\circ}$ et $90^{\circ}$ ont été collées au centre de la surface utile de chaque éprouvette. Avant la fissuration du matériau, ces jauges permettent de mesurer et vérifier l'état de déformation biaxiale en un point de la partie centrale $\left(30 \times 30 \mathrm{~mm}^{2}\right)$. Une fois la fissure apparue, on perd les informations des jauges. L'évolution de la fissuration est alors suivie à l'aide d'une analyse d'images. Ces mesures optiques réalisées suivant l'axe vertical permettent de mesurer et vérifier l'état de déformation élastique et de suivre en temps réel l'évolution de la fissuration en tout point de la zone utile $\left(100 \times 100 \mathrm{~mm}^{2}\right)$.

\subsection{Chargement}

Le chargement appliqué est dit "quasi-instantané", c'est-à-dire que la durée de celui-ci est suffisamment courte pour qu'on puisse considérer comme négligeables les effets de vieillissement, de fluage ou de relaxation. La vitesse de chargement de chaque vérin est toujours constante (environ $40 \mu \mathrm{m} / \mathrm{min}$ ). Les déplacements sont interrompus par des paliers tel que l'on voit sur la figure 11, ces paliers dans le temps permettent de réaliser des acquisitions d'images de la zone utile à chaque pas.

\subsection{Acquisitions}

De façon automatique on acquiert toutes les secondes les valeurs de déformation des jauges, les efforts et les déplacements des vérins de la machine. Manuellement 
les images de la zone utile son acquises à chaque niveau de déplacement défini par paliers.

\subsubsection{L'acquisition d'images}

Le dispositif utilisé comprend une caméra CCD Kodak (Charge Coupled Device), couplée à une carte d'acquisition permettant de stocker les images prises sur le disque dur d'un ordinateur. Les images de 1360 x 1024 pixels (images .TIF, signal codé sur 12 bits, 4096 niveaux de gris) correspondent à la zone utile de l'éprouvette (environ $100 \times 100 \mathrm{~mm} 2$ ) (Figure 11) à chaque palier du chargement. Afin de créer une texture aléatoire, la zone utile de l'éprouvette est recouverte d'un mouchetis artificiel fabriqué à l'aide de peinture noire et blanche. Le champ de déplacement plan est obtenu par corrélation entre deux images choisies.

\subsubsection{CORRELI $2 D$ : mesure de déplacements par corrélation d'images}

CORRELI 2D est un outil de dépouillement d'images développé au LMT par François Hild [Hild et al, 1999], et a été programmé sur plate-forme Matlab avec un environnement graphique très convivial. Son utilisation présente plusieurs avantages pour notre étude. Tout d'abord, il permet de contrôler de façon rapide l'homogénéité du champ de déplacement dans toute la zone utile de l'éprouvette et éventuellement de corriger le chargement en conséquence. Il permet aussi de mesurer les déformations de cette zone avec bonne précision. Une fois la fissure amorcée, les informations obtenues à partir des jauges cessent, c'est alors que l'intercorrélation d'images prend le relais et permet de suivre l'évolution des fissures. Finalement et grâce au fait que cette méthode permet de faire des analyses locales, les déplacements peuvent être quantifiés en tout point de la surface observée.

D'une façon générale, la méthode d'intercorrélation consiste à comparer deux images de la surface observée pour deux niveaux de chargement différents, la première image est alors dite de "référence" et la deuxième "déformée". Une section de l'image de référence est sélectionnée et identifiée par les valeurs de gris de chaque pixel, et on recherche dans l'image déformée la section de pixels la plus semblable à la précédente en termes de niveaux de gris.

L'algorithme d'intercorrélation employé s'avère efficace et rapide grâce à l'utilisation de la Transformée Rapide de Fourier (TFR). Des déformations variant de $\varepsilon=10^{-4}$ à $\varepsilon=10^{-1}$ sont mesurables avec une très bonne précision.

\section{Résultats expérimentaux}

Les résultats d'un essai sont montrés par la suite, notamment ceux correspondant aux efforts, déformations et champ de déplacement. La figure 12 montre l'évolution du rapport d'efforts F1 / F2 ; on aperçoit sur ce graphique que les efforts sont 
symétriques jusqu'à une valeur maximale de $48 \mathrm{kN}$, après cette valeur une chute d'effort apparaît indiquant l'amorçage d'une première fissure.

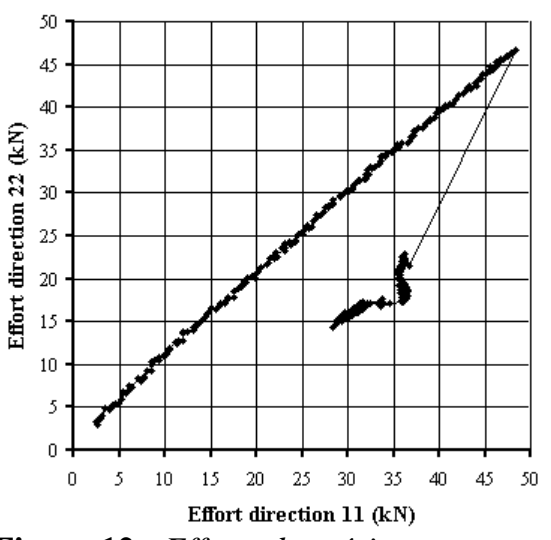

Figure 12 : Efforts des vérins

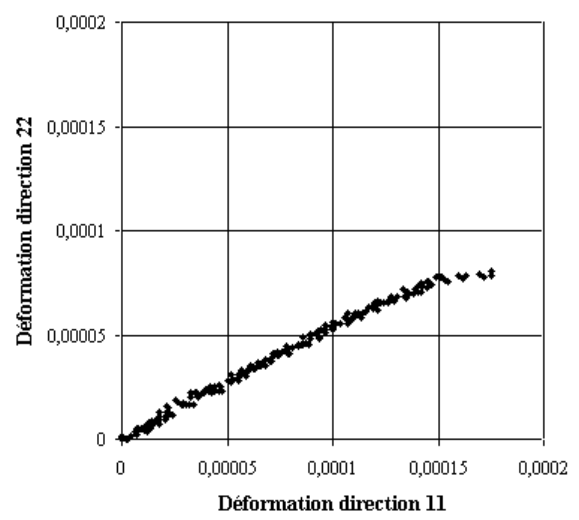

Figure 13 : Déformations jauges

Sur la figure 13 sont montrées les déformations mesurées par les jauges avant fissuration. On note que, malgré la symétrie d'efforts obtenue, les déformations sont dissymétriques et atteignent des valeurs maximales différentes $(\varepsilon 11=0,00017$ et $\varepsilon 22=00008$ ). Ceci pourrait s'expliquer par une dissymétrie des raideurs de liaisons éprouvette - machine.

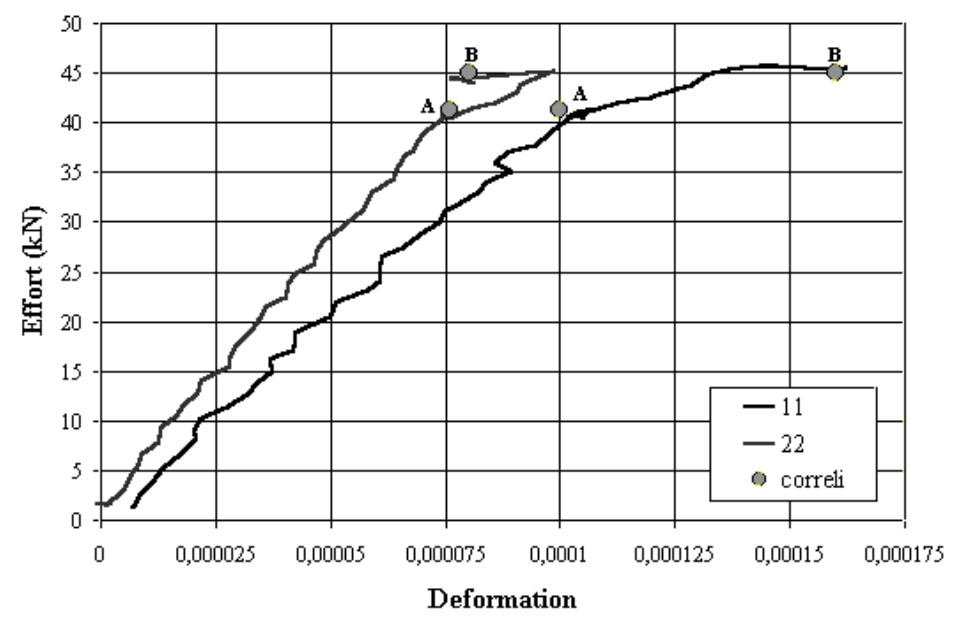

Figure 14 : Courbe Effort - Déformation

L'évolution du champ de déplacement est visualisée dans le post - processeur de CORRELI 2D, la mesure des déformations y est aussi possible. Une façon de percevoir les performances de CORRELI 2D pour la mesure de déformations, 
consiste à calculer les déformations dans la zone correspondant aux jauges. La figure 14 montre les courbes Effort - Déformation dans les deux directions principales. Les points A et B sur les courbes correspondent aux déformations mesurées avec CORRELI 2D à partir des images A et B prises dans les paliers de $41 \mathrm{kN}$ et $45 \mathrm{kN}$ respectivement et corrélées toutes les deux avec une image de référence prise avant chargement. On constate alors un bon accord des résultats issus de l'outil d'analyse d'images avec la réalité.

La figure 15 montre le champ de déplacement obtenu de l'image A correspondant à un effort de $41 \mathrm{kN}$ avec des déformations dans la zone de jauges $\varepsilon 11=0,0001$ $\varepsilon 22=0,000076$. Le carré au centre de la figure indique la zone où les jauges sont placées. La figure 16 montre le champ de déplacement obtenu de l'image $\mathrm{B}$, dernière image prise avant fissuration, au moment où la valeur de l'effort était de $45 \mathrm{kN}$ et les déformations calculées étaient $\varepsilon 11=0,00017$ et $\varepsilon 22=0,00008$.

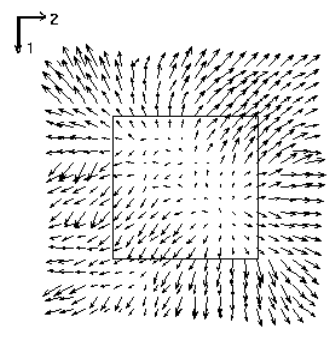

Figure 15 :Déplacements image A

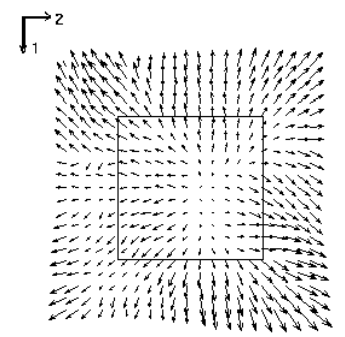

Figure 16 :Déplacements image $B$

Afin d'essayer de visualiser à partir des images $\mathrm{A}$ et $\mathrm{B}$ la zone où la première fissure pourrait s'amorcer, nous avons corrélé ces deux images en prenant l'image A comme image de référence. Le résultat de ce calcul est donné par la figure 17 où l'on constate une perturbation du champ de déplacement dans le coin inférieur gauche du champ de déplacement. La figure 18 montre l'évolution des déplacements toute suite après fissuration, on voit que la fissure s'est amorcée justement dans la zone perturbée (figure 18).

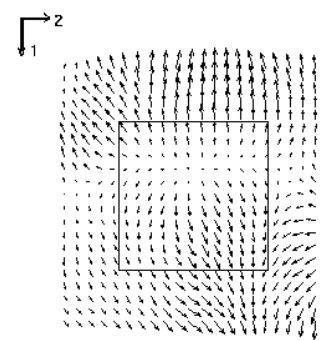

Figure 17 :Image A corrélée avec B

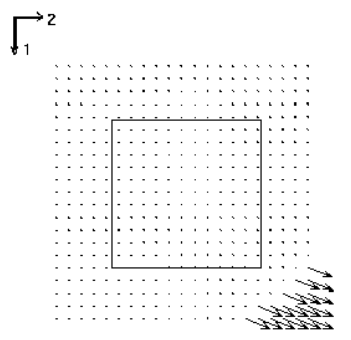

Figure 18 :Déplacements post-fissuration 
Les déformations maximales mesurées avant fissuration $(\varepsilon=0,00017)$ sont inférieures à celles mesurés en traction simple pour ce même type de matériau $(\varepsilon=0,00025)$ [BEH 96]. Cependant ces déformations sont supérieures à celles du béton ordinaire soumis au même type de sollicitation $(\varepsilon=0,00008)$ [KUP 69] et à celles du béton hautes performances $(\varepsilon=0,00012)$ [HUS 00].

Dans la figure 19, les valeurs de la contrainte maximale avant fissuration issues des essais sont comparées à celles de la littérature et permettent de proposer un critère de fissuration pour ce type de matériau. Une image de fin d'essai est donnée sur la figure 20 .
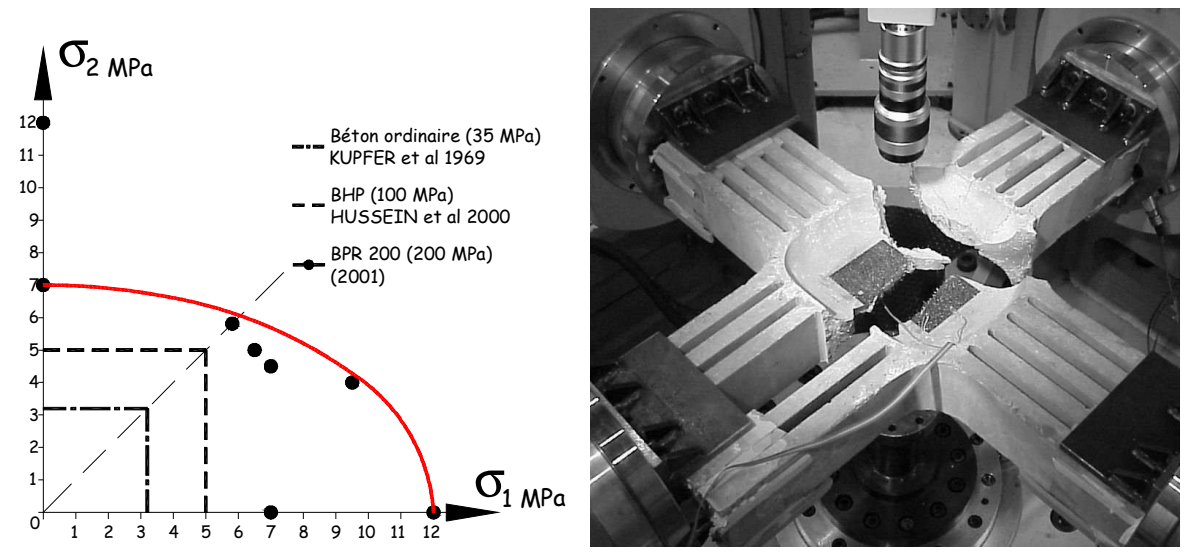

Figure 19 : Valeurs de contrainte Figure 20: Image de fin d'essai maximale avant fissuration

\section{Conclusions}

Un essai de traction biaxiale sur éprouvette cruciforme en Béton de Poudres Réactives armé de fibres courtes d'acier a été conçu et réalisé. Nous avons porté nos efforts sur la préparation et l'optimisation de l'éprouvette. Une méthode fiable et robuste d'intercorrélation d'images a été utilisée avec succès. Ce travail en amont nous a permis de réduire le nombre d'essais et d'obtenir des résultats expérimentaux pertinents. Nous proposons d'ailleurs un critère de fissuration pour ce type de matériau dans une configuration de sollicitations particulières.

\section{Remerciements}

Les auteurs tiennent à remercier le Consejo Nacional de Ciencia y Tecnologia du Mexique pour son soutien économique, M. Mazars, M. Calloch et M. Hild pour leur contribution scientifique à ce travail. 


\section{Bibliographie}

[BEH 96] Behloul, M., « Analyse et modélisation du comportement d'un matériau à matrice cimentaire fibrée à ultra hautes performances (Bétons de Poudres Réactives) Du matériau à la structure », thèse de doctorat, Ecole Normale Supérieure de Cachan.

[COG 97] Cognard, J.Y., Feuardent, V., Virely, J.M., « Optimisation of a structure for biaxial mechanical tests ». Integrated Design and Manufacturing in Mechanical Engineering, Chedmail, P., éditeur, Netherlands, 495-504

[HIL 99] Hild, F., Périé J.N., Coret, M., « Mesure de champs de déplacements 2D par corrélation d'images numériques : CORRELI 2D », Rapport Interne LMT No. 230, décembre 1999.

[HUS 00] Hussein, A., Marzouk, H., « Behavior of High-Strength Concrete under Biaxial Stresses ». ACI Materials Journal, january-february 2000, 27-36.

[KUP 69] Kupfer, H., Hilsdorf, H., Rusch, H., « Behavior of Concrete under Biaxial Stresses ». ACI Journal, Proceedings, 66, 8, 656-666.

[MOR 85] Morrison, C.J., « Biaxial testing using cruciform specimens ». Techniques for Multiaxial Creep Testing, Gooch, D.J., How, I.M., éditeurs, Elsevier Science, 111-127.

[RIC 95] Richard, P., Cheyrezy, M., « Les Bétons de Poudres Réactives ». Annales de l'ITBTP, 532, 85-102. 